\title{
Escovagem de dentes em ambiente escolar e cárie dentária: um estudo de coorte
}

Ana Paula Rodrigues, * Patrícia Reis**

\section{RESUMO}

Objectivos: Com este trabalho pretendeu-se avaliar a protecção conferida pela escovagem de dentes na escola na redução das cáries dentárias na infância.

Tipo de estudo: Coorte histórico

Local: Escolas públicas de Ovar, Portugal.

População: Crianças residentes no concelho de Ovar a frequentar o ensino pré-escolar público.

Métodos: A partir da informação disponível das avaliações de saúde oral efectuadas no âmbito do Programa Nacional de Promoção da Saúde Oral foram estudadas 62 crianças de 4 anos de idade divididas em dois grupos: crianças que realizaram escovagem de dentes na escola durante 21 meses e crianças que não realizaram escovagem de dentes na escola no mesmo período. Ao fim de 29 meses de seguimento, comparou-se entre os dois grupos: o número de novas cáries, o índice de dentes cariados, perdidos e obturados nos dentes deciduais (cpod) e o índice de dentes cariados, perdidos e obturados nos dentes definiti$\operatorname{vos}(C P O D)$.

Resultados: O risco de cárie dentária foi, ao fim de 29 meses, de 50,0\% no grupo de crianças que realizaram escovagem de dentes e de $62,5 \%$ no grupo de crianças que não efectuou escovagem de dentes na escola, correspondendo a um risco relativo de 0,75 (IC 95\%: 0,42-1,33). O índice de cpod no grupo que efectuou escovagem de dentes foi 1,27 (IC 95\%: 0,69-1,94) e no grupo que não efectuou escovagem foi 2,28 (IC 95\%: 1,39-3,19), $P=0,078$. O índice CPOD no grupo que efectuou escovagem foi 0,37 (IC 95\%: 0,08-0,71) e no grupo que não efectuou escovagem foi 0,63 (IC 95\%: 0,29 - 1,03), $p=0,315$.

Conclusões: Não foi encontrada associação estatisticamente significativa entre escovagem de dentes na escola e redução do risco de cárie dentária, embora para tal possa ter contribuído o reduzido tamanho do grupo de estudo.

Palavras-Chave: Escovagem de Dentes; Cárie; Estudo de Coorte.

\section{INTRODUÇÃO}

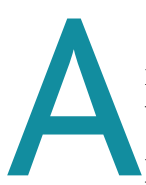

cárie dentária é uma das doenças crónicas mais comuns durante a infância, afectando o bem-estar e o desenvolvimento infantil. ${ }^{1} \mathrm{~A}$ prevalência de cárie dentária em Portugal aos 6 anos é de $49 \%$, aumentando até aos $72 \%$ aos 15 anos de idade. ${ }^{2} \mathrm{O}$ índice de dentes cariados, perdidos e obturados na dentição temporária (cpod) e definitiva (CPOD) aos 6 anos é, respectivamente, de 0,07 e 2,10, aumentando este último, até aos 3,04 aos 15 anos. ${ }^{2}$

No concelho de Ovar, onde o presente estudo foi

*Médica de Saúde Pública; Centro de Saúde de Oliveira do Bairro, Unidade de Saúde Pública, Agrupamento de Centros de Saúde do Baixo Vouga I.

**Higienista oral; Centro de Saúde de Ovar, Unidade de Saúde Pública, Agrupamento de Centros de Saúde do Baixo Vouga III. efectuado, com cerca de 9.281 residentes menores de 14 anos, ${ }^{3}$ a proporção de crianças livres de cáries aos 6 e 13 anos de idade foi, em 2009, de, respectivamente, $41,9 \%$ e 45,5\%. ${ }^{4}$ Os índices CPOD aos 6 e 13 anos foram de 0,2 e 1,9, sendo o cpod aos 6 anos de 2,5. ${ }^{4}$

A prevenção primária da cárie dentária em idades precoces assenta na utilização de fluoretos tópicos, ${ }^{5-10}$ na redução do consumo de açúcares e em fluoretos sistémicos em crianças com elevado risco de cárie, ${ }^{10}$ estando demonstrado que a escovagem de dentes com dentífrico fluoretado é a estratégia universal mais custo-efectiva e que a escovagem bidiária é mais eficaz que a restrição de açúcares na redução da cárie dentária. ${ }^{11}$

Actualmente, recomenda-se que sejam efectuadas, pelo menos, duas escovagens diárias com dentífrico 
fluoretado de 1.000 a $1.500 \mathrm{ppm}$, devendo uma das escovagens ser feita antes do deitar. ${ }^{7,12,13} \mathrm{Em}$ Portugal, apenas $50 \%$ das crianças de 6 anos de idade escovam os dentes pelo menos duas vezes por dia, sendo que na região centro apenas $30 \%$ das crianças têm este hábito. ${ }^{2}$

A estratégia europeia e as metas da Organização Mundial de Saúde apontam para que no ano 2020 pelo menos $80 \%$ das crianças com 6 anos estejam livres de cárie, referindo o contexto escolar como ideal para desenvolver programas de promoção da saúde oral durante a infância, ${ }^{2}$ estando também documentada uma melhor saúde oral nas crianças que frequentam escolas promotoras de saúde, ${ }^{14}$ embora se saliente que a efectividade destes programas é influenciada pelo nível de escolaridade dos pais, ${ }^{15}$ pela percepção dos pais sobre a sua própria saúde oral ${ }^{16,17}$ e pelo contexto social das famílias. ${ }^{18}$ Em Portugal, muitos dos programas escolares de promoção da saúde oral assentam na realização de sessões de educação para a saúde para crianças e, em alguns casos, também para pais, não tendo sido demonstrada a eficácia dessas intervenções na redução do número de cáries em diversos estudos internacionais. ${ }^{5,19,20}$ Existe, no entanto, evidência da melhoria de comportamentos e redução da cárie dentária com intervenções com pais e crianças que visam a divulgação de conhecimentos de saúde oral ${ }^{21-23} \mathrm{e}$ a supervisão da escovagem em ambiente escolar. ${ }^{24-32}$ Embora não estejam publicados estudos que demonstrem a efectividade desta estratégia na população portuguesa, ela tem sido recomendada pela Direcção-Geral da Saúde (DGS). No entanto, a prática tem mostrado alguma resistência por parte dos educadores na implementação da escovagem de dentes em meio escolar, ${ }^{33}$ pelo que nos parece pertinente avaliar o seu efeito protector, tendo em conta os demais factores que influenciam a saúde oral em Portugal.

No concelho de Ovar têm sido desenvolvidos alguns esforços para implementar a escovagem de dentes em ambiente escolar, garantindo, deste modo, pelo menos uma escovagem de dentes diária com supervisão de um adulto e contribuindo para a aprendizagem precoce deste hábito de higiene oral.

Com este estudo exploratório pretende-se avaliar o efeito protector da escovagem de dentes na escola no contexto português, usando os registos dos rastreios dentários efectuados no âmbito do Programa Nacional de Promoção da Saúde Oral (PNPSO) entre 2006 e 2009.

\section{MÉTODOS}

Efectuou-se um estudo de coorte, histórico, usando como fonte de informação a ficha do rastreio oral usada no PNPSO. A partir dos dados registados em Setembro de 2006, no âmbito do PNPSO, seleccionaram-se dois grupos de crianças residentes no concelho de Ovar a frequentar o ensino pré-escolar público:

1. Grupo exposto: todas as crianças de 4 anos de idade livres de cárie no início da observação que iniciaram nesse ano a escovagem de dentes diária na escola (total de 30 crianças). A exposição foi definida como uma escovagem de dentes diária na escola, após o almoço, durante o período lectivo, usando um dentrífico fluoretado (conteúdo de flúor de 1000 a $1500 \mathrm{ppm}$ ). O período de exposição correspondeu a 21 meses (Setembro de 2006 a Junho de 2008).

2. Grupo não-exposto: todas as crianças de 4 anos de idade, livres de cárie no início da observação que não iniciaram a escovagem de dentes na escola $\mathrm{e}$ que frequentavam a mesma escola do grupo dos expostos ou a escola mais próxima (total de 32 crianças).

Para ambos os grupos, a escovagem ou não escovagem de dentes na escola dependeu da decisão da educadora de cada turma.

O tempo total de seguimento foi de 29 meses, tendo sido realizadas duas observações: a primeira em Setembro de 2006 (no início do estudo) e a segunda em Fevereiro de 2009, isto é, 8 meses após o final da exposição à escovagem de dentes na escola. Foram consideradas como perdas ao seguimento as crianças que abandonaram o PNPSO até Junho de 2008 ou que não tenham realizado a segunda observação clínica em Fevereiro de 2009. (Figura 1).

Uma vez que foram incluídas todas as crianças livres de cáries que escovaram os dentes na escola diariamente e que se desconhecia a diferença de risco esperada entre os dois grupos, não foi calculado o tamanho do grupo de estudo. Posteriormente, foi calculada a potência estatística do estudo e o tamanho de amostra necessário para detectar a diferença de risco estimada.

A ficha de registo de saúde oral, usada como única fonte de informação, continha a seguinte informação: 


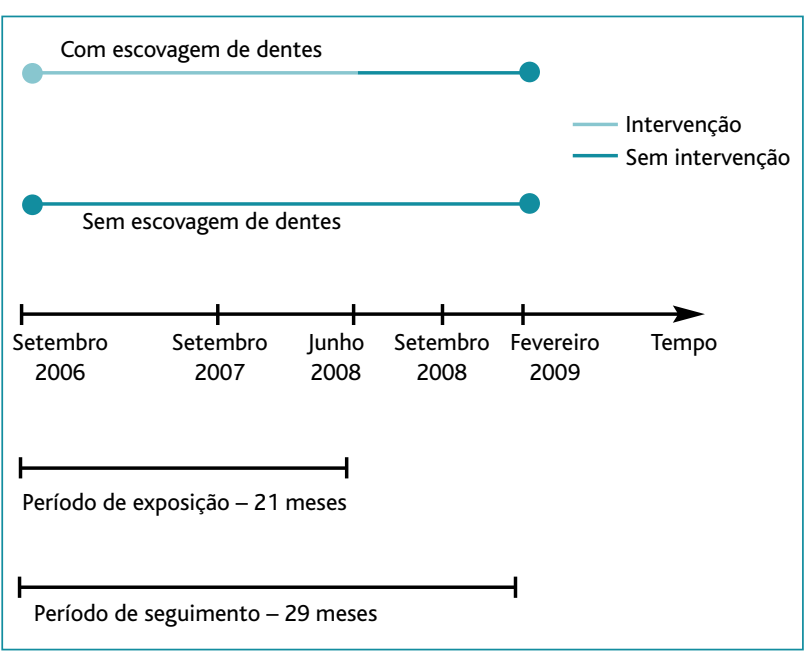

Figura 1. Modelo conceptual do estudo.

- Dados demográficos: nome, idade, escola;

- Dados de saúde oral: número de dentes temporários, número de dentes definitivos, identificação dos dentes cariados, perdidos e obturados na dentição temporária e definitiva.

Para efeitos do presente estudo foram considerados:

1. Dentes cariados, os dentes que apresentavam perda de substância, uma superfície perdida, uma cavidade clinicamente visível, uma cavidade com amolecimento de fundo ou uma parede amolecida, uma obturação com uma cárie primária ou recidiva e uma obturação provisória.

2. Dentes obturados, aqueles que apresentavam restauração com amálgama ou restauração com compósito.

3. Dentes perdidos, os dentes que foram perdidos ou extraídos devido a cárie.

4. Não foram considerados, para efeito do presente estudo, os dentes não presentes na cavidade oral (dentes não erupcionados, ausência congénita de dentes ou extraídos por outras razões que não a cárie dentária) e os dentes restaurados por outras razões que não a cárie dentária.

Todas as observações clínicas registadas foram realizadas na sala de aula, com luz natural, pela mesma higienista oral, previamente treinada, de acordo com os critérios definidos pela DGS para o programa nacional de saúde oral. ${ }^{2} \mathrm{O}$ diagnóstico foi efectuado por observação directa. Nas situações não diferenciáveis à observação, por exemplo por não ser possível identificar o motivo da extracção dentária, foi considerado o estado dos dentes adjacentes e a informação fornecida pelas crianças.

Tendo por base os critérios explicitados, foram identificadas todas as crianças que apresentaram cáries dentárias em Setembro de 2006 (início do estudo) e em Fevereiro de 2009 (final do estudo), bem como o número de cáries, dentes perdidos e obturados por cárie apresentado por cada criança no final do estudo.

Em seguida, calculou-se o risco de cárie correspondente ao período de seguimento (risco cumulativo em 29 meses), os índices de cpod e o CPOD para cada grupo no momento da segunda observação. Como medida de risco foi usado o risco relativo. Para as estimativas pontuais de cada parâmetro foram calculados os intervalos de confiança a 95\%. Apesar do reduzido tamanho da amostra, tendo em conta o teorema do limite central, aplicou-se o teste do $\chi^{2}$ para comparação de proporções e o teste $t$ de Student (corrigido nas situações de desigualdade das variâncias) para comparação de médias. Foi definido um nível de significância de 0,05 .

Para a análise estatística utilizou-se o programa informático Statistical Programme for Social Sciences (SPSS ${ }^{\circledast}$ ), na sua versão v.20.0.0.

\section{RESULTADOS}

\section{Caracterização do grupo de estudo}

O grupo de estudo foi constituído por 62 crianças, das quais $30(48 \%)$ realizaram escovagem de dentes regular durante 21 meses. (Quadro I) No início do estudo (Setembro de 2006), todas as crianças tinham 4 anos e ambas as coortes eram homogéneas em relação ao sexo $(p=0,779)$. Não foram consideradas outras variáveis potencialmente importantes para o estudo, como o nível socioeconómico, a escolaridade dos pais, o número de escovagens diárias, a alimentação e a exposição ao flúor, pois estas variáveis não se encontravam registadas na ficha individual do PNPSO.

Nenhuma criança foi perdida para o estudo ao longo do tempo de seguimento, pelo que a constituição do grupo não se alterou durante os 29 meses de seguimento e o tempo de exposição à escovagem de dentes em ambiente escolar foi de 21 meses para todas as crianças. 


\begin{tabular}{|c|c|c|}
\hline \multicolumn{3}{|c|}{$\begin{array}{l}\text { QUADRO I. Distribuição das coortes de estudo de } \\
\text { acordo com sexo dos participantes. }\end{array}$} \\
\hline Coorte de estudo & Total & $\begin{array}{l}\text { Masculino } \\
\text { n (\%) }\end{array}$ \\
\hline Com escovagem & 30 & $17(57 \%)$ \\
\hline Sem escovagem & 32 & 17 (53\%) \\
\hline Total & 62 & $34(55 \%)$ \\
\hline
\end{tabular}

$p=0,779$

\section{Risco de cárie e índices de cárie}

No final dos 29 meses de seguimento foram identificadas 35 crianças com cáries dentárias, a que corresponde um risco de cárie dentária de $56,5 \%$ (IC $95 \%$ : $43,8 \%-69,2 \%$ ). Na coorte de expostos, surgiram 15 novos casos de cáries dentárias (50,0\%; IC 95\%: 31,0\% $69,0 \%)$ e na coorte de não expostos surgiram 20 novos casos de cárie (62,5\%; IC 95\%: 44,8\% - 80,2\%) (Quadro II).

No grupo de crianças expostas à escovagem de dentes na escola, os índices de cpod e CPOD foram, respectivamente, 1,27 (IC 95\%: 0,69 - 1,94) e 0,37 (IC 95\%: 0,08-0,71) (Quadro III). O número máximo de dentes cariados por criança foi de 6 . No grupo de crianças não expostas à escovagem de dentes na escola os índices de cpod e CPOD foram, respectivamente, 2,28 (IC 95\%: 1,39 - 3,19) e 0,63 (IC 95\%: 0,29 - 1,03) (Quadro III). O número máximo de dentes cariados por criança foi 10 .

$O$ risco relativo (RR) entre os 2 grupos foi de 0,75 (IC 95\%: 0,42-1,33). Comparando os índices de cáries entre os dois grupos não foram encontradas diferenças estatisticamente significativas.

\section{DISCUSSÃO}

Ao contrário do evidenciado em outros estudos, ${ }^{23-}$ ${ }^{31}$ não foi possível demonstrar um menor risco de desenvolvimento de cárie dentária no grupo de crianças que realizou escovagem de dentes na escola (RR de 0,75; IC 95\%: 0,42-1,33), o que pode estar relacionado com o reduzido tamanho do grupo de estudo e com a consequente limitada potência do estudo $(\beta=0,62)$.

A utilização de dados do rastreio de saúde oral, colhidos com outro fim que não o estudo da protecção da escovagem de dentes, e a escolha não aleatória do

\begin{tabular}{|c|c|c|}
\hline \multicolumn{3}{|c|}{$\begin{array}{l}\text { QUADRO II. Crianças com cárie dentária em cada uma } \\
\text { das coortes no final do período de seguimento } \\
\text { (Fevereiro de 2009). }\end{array}$} \\
\hline Coorte de estudo & Total & $\begin{array}{c}\text { Com cárie } \\
\text { n (\%) }\end{array}$ \\
\hline Com escovagem & 30 & 15 (50\%) \\
\hline Sem escovagem & 32 & $20(62 \%)$ \\
\hline Total & 62 & $35(56 \%)$ \\
\hline
\end{tabular}

grupo de estudo impossibilitou o controlo de outras variáveis de interesse e potencialmente confundidoras dos resultados, como os hábitos alimentares, o nível socioeconómico, o nível de exposição a outras fontes de flúor e o número de escovagens diárias de cada criança. Estas variáveis, cuja distribuição poderá ter sido distinta nos dois subgrupos em estudo, podem ter mascarado uma verdadeira associação entre escovagem de dentes na escola e cárie dentária.

O diagnóstico por observação directa (em sala de aula e sem recurso a meios de diagnóstico) e a utilização de informações fornecidas pelas crianças podem ter introduzido um viés de medição. A utilização da mesma metodologia, previamente protocolada, pretendeu minimizar o impacto deste viés. Não se tratando de um viés selectivo, isto é, com efeito apenas sobre um dos subgrupos de estudo, é plausível que as comparações entre os dois subgrupos (expostos/não expostos) possam não ter sido tão afectadas por esse motivo.

Além do referido, o efeito da escovagem de dentes em ambiente escolar pode estar aqui diminuído pelo fac-

QUADRO III. Índice de cáries no final do período de
observação (Fevereiro 2009).
\begin{tabular}{l|c|c|c|c} 
Coorte de estudo & cpod & IC 95\% & CPOD & IC $95 \%$ \\
\hline Com escovagem & 1,27 & $(0,69-1,94)$ & 0,37 & $(0,08-0,71)$ \\
\hline Sem escovagem & 2,28 & $(1,39-3,19)$ & 0,63 & $(0,29-1,03)$
\end{tabular}

IC 95\%: intervalo de confiança a 95\%; cpod: índice de dentes temporários cariados, perdido e obturados ( $p=0,078)$; CPOD: índice de dentes definitivos cariados, perdidos e obturados $(p=0,315)$ 
to de a segunda observação ter ocorrido 8 meses após o final da exposição. Durante este período nenhuma criança escovou os dentes na escola pelo que, atendendo ao mecanismo de acção da escovagem na protecção contra as cáries dentárias, o risco de cárie após o período de exposição poderá ter sido superior ao verificado anteriormente, circunstância que afectaria sobretudo a medição do efeito no grupo exposto, isto é: se a observação fosse feita imediatamente após o fim do período de exposição ao flúor, o grupo que escovou os dentes talvez apresentasse um menor número de cáries.

Salienta-se a elevada incidência de cárie dentária neste grupo etário (56,5\%) em tão curto intervalo de tempo (29 meses), o que sugere a pertinência de intervenções de prevenção primária e secundária que visem reduzir o aparecimento de novas cáries, seu diagnóstico e tratamento precoce, assim como o desenvolvimento de estudos que demonstrem a adequação destas estratégias de promoção da saúde oral, nomeadamente da escovagem de dentes na escola, à população portuguesa.

Apesar das limitações referidas, os resultados obtidos neste estudo exploratório poderão ser úteis ao desenho de estudos mais robustos, podendo a proporção de crianças com cáries aqui estimada para cada um dos grupos ser usada como pressuposto necessário para o cálculo amostral.

Assim, para detectar uma diferença entre os grupos de $6,5 \%$ na incidência de cáries, com uma potência de $90 \%$ e um erro do tipo alfa de $5 \%$, seria necessária uma amostra de 1267 crianças em cada um dos grupos. Igualmente importante será alongar o mais possível o período de observação, usar metodologias de emparelhamento entre expostos e não expostos, repetir as observações em vários pontos do período de seguimento e controlar o maior número possível de factores de confundimento.

\section{AGRADECIMENTOS}

A Pedro Serrano e lolanda Duarte pelas amáveis sugestões e comentários.

\section{REFERÊNCIAS BIBLIOGRÁFICAS}

1. Arora A, Scott JA, Bhole S, Do L, Schwarz E, Blinkhorn AS. Early childhood feeding practices and dental caries in preschool children: a multi-centre birth cohort study. BMC Public Health 2011 Jan 12; 11: 28.

2. Direcção-Geral de Saúde (DGS). Estudo de prevalência de doenças orais em Portugal. Lisboa: DGS; 2008.

3. Instituto Nacional de Estatistica (INE). Estatísticas territoriais. Lisboa: 2011.Disponível em: http://www.ine.pt/xportal/xmain?xpid= INE\&xpgid=ine_unid_territorial $\&$ menuBOU|=13707095\&contexto $=u t \&$ selTab=tab3 [acedido em 15/10/2012].

4. Queirós AR, Reis P. Boca Saudável Sorriso Feliz. Ovar:Agrupamento de Centros de Saúde Baixo Vouga III; 2009.

5. Twetman S. Prevention of early childhood caries (EEC): review of literature published 1998-2007. Eur Arch Paediatr Dent 2008 Mar; 9 (1): 12-8.

6. de Sousa M da L, Wagner M, Sheiham A. Caries reductions related to the use of fluorides: a retrospective cohort study. Int Dent J 2002 Oct; 52 (5): 315-20.

7. Walsh T, Worthington HV, Glenny AM, Appelbe P, Marinho VC, Shi X. Fluoride toothpastes of different concentrations for preventing dental caries in children and adolescents. Cochrane Database Syst Rev 2010 JN 20; (1): CD007868.

8. Twetman S. Caries prevention with fluoride toothpaste in children: an update. Eur Arch Paediatr Dent 2009 Sep; 10 (3): 162-7.

9. Newbrun E. Topical fluorides in caries prevention and management: a North American perspective. J Dent Educ 2001 Oct; 65 (10): 1078-83.

10. Tinanoff N, Kanellis MJ, Vargas CM. Current understanding of the epidemiology mechanisms, and prevention of dental caries in preschool children. Pediatr Dent 2002Nov-Dec; 24 (6): 543-51.

11. Gibson S,Williams $S$. Dental caries in pre-school children: associations with social class, toothbrushing habit and consumption of sugars and sugarcontaining foods. Further analysis of data from the National Diet and Nutrition Survey of children aged 1.5-4.5 years. Caries Res 1999; 33 (2):10113.

12. Direção-Geral de Saúde (DGS). Programa Nacional de Promoção da Saúde Oral. Lisboa: DGS; 2005.

13. Ammari AB, Bloch-Zupan A,Ashley PF. Systematic review of studies comparing the anti-caries efficacy of children's toothpaste containing 600 ppm of fluoride or less with high fluoride toothpastes of 1,000 ppm or above. Caries Res 2003 Mar-Apr; 37 (2): 85-92.

14. Moysés ST, Moysés SJ,Watt RG, Sheiham A. Associations between helath promoting schools' policies and indicators of oral health in Brazil. Health Promot Int 2003 Sep; 18 (3): 209-18.

15. Tomita NE, Fagote BF. Programa educativo em saúde bucal para pacientes especiais. Odontol Sociedade 1999; 1(1/2): 45-50.

16. Passalacqua A, Reeves AO, Newton T, Hughes R, Dunne S, Donaldson N, et al. An assessment of oral health promotion programmes in the United Kingdom. Eur J Dent Educ 2012 Feb; 16 (1): e19-26.

17. Lapresa LB, Barbero BS, Arizaleta LH. Prevalencia de hábitos bucodentales saludables en la población infanto-juvenil residente en Espana. An Pediatr (Barc) 2012 Mar; 76 (3): 140-7.

18. Amalia R, Schaub RM, Widyanti N, Stewart R, Groothoff JW. The role of school-based dental programme on dental caries experience in Yogyakarta Province, Indonesia. Int J Paediatr Dent 2012 May; 22 (3): 203-10.

19. Esfahanizadeh N. Dental health education programme for 6 -year-olds: a cluster randomised controlled trial. Eur J Paediatr Dent 2011 Sep; 12 (3): 167-70.

20. Ismail Al, Ondersma S, Jedele JM, Little RJ, Lepkowski JM. Evaluation of a brief tailored motivational intervention to prevent early childhood caries. Community Dent Oral Epidemiol 2011 Oct; 39 (5): 433-48. 
21. Wennhall I, Matsson L, Schröder U, Twetman S. Outcome of an oral health outreach programme for preschool children in a low socioeconomic multicultural area. Int J Paediatr Dent 2008 Mar; 18 (2): 84-90.

22. Davies GM, Duxbury JT, Boothman NJ, Davies RM, Blinkhorn AS. A staged intervention dental health promotion programme to reduce early childhood caries. Community Dent Health 2005 Jun; 22 (2): 118-22.

23. Pine CM, McGoldrick PM, Burnside G, Curnow MM, Chesters RK, Nicholson J, et al. An intervention programme to establish regular toothbrushing: understanding parents' beliefs and motivating children. Int Dent J 2000; Suppl Creating A Successful: 312-23.

24. Jackson RJ, Newman HN, Smart GJ, Stokes E, Hogan JI, Brown C, et al. The effects of a supervised toothbrushing programme on the caries increment of primary school children, initially aged 5-6 years. Caries Res 2005 MarApr; 39 (2):108-15.

25. Curnow MM, Pine CM, Burnside G, Nicholson JA, Chesters RK, Huntington $\mathrm{E}$. A randomised controlled trial of the efficacy of supervised toothbrushing in high-caries-risk children. Caries Res 2002 Jul-Aug; 36 (4): 294 300 .

26. Rong WS, Bian JY, Wang WJ, Wang JD. Effectiveness of an oral health education and caries prevention program in kindergartens in China. Community Dent Oral Epidemiol 2003 Dec; 31(6): 412-6.

27. Al-Jundi SH, Hammad M, Alwaeli $H$. The efficacy of a school-based caries preventive program: a 4-year study. Int J Dent Hyg 2006 Feb; 4 (1): 30-4

28. Andruskeviciene V, Milciuviene S, Bendoraitiene E, Saldunaite K, Vasiliauskiene I, Slabsinskiene $E$, et al. Oral health status and effectiveness of caries prevention programme in kindergartens in Kaunas city (Lithuania). Oral Health Prev Dent 2008; 6(4): 343-8.

29. Moorhead JE, Conti AJ, Marks RG, Cancro LP. The effect of supervised brus- hing on caries inhibition in school age children. J Clin Dent 1991; 2(4):97102.

30. Hölttä P, Alaluusua S. Effect of supervised use of a fluoride toothpaste on caries incidence in pre-school children. Int J Paediatr 1992 Dec; 2 (3): 1459.

31. Kerebel LM, Le Cabellec MT, Daculsi G, Kerebel B. Report on caries reduction in French schoolchildren 3 years after the introduction of a preventive program. Community Dent Oral Epidemiol 1985 Aug; 13 (4): 201-4.

32. Milgrom P, Tut OK. Evaluation of Pacific Islands Early Childhood Caries Prevention Project: Republic of the Marshall Islands. J Public Health Dent 2009 Summer; 69 (3): 201-3.

33. Direcção Geral da Saúde (DGS). Projecto SOBE. DGS; 2012. Disponível em: http://www.sobe.pt/epages/2933120726.sf/pt_PT/?ObjectPath=/Shops/ 2933-120726/Categories/Familias_Alunos/Carie_Dentaria [acedido em 15/10/2012].

\section{CONFLITO DE INTERESSES}

As autoras declaram não possuir qualquer tipo de conflito de interesses.

\author{
ENDEREÇO PARA CORRESPONDÊNCIA \\ Ana Paula Rodrigues \\ Centro de Saúde de Oliveira do Bairro, Rua do Hospital n 20 \\ 3770-210 Oliveira do Bairro \\ E-mail: ana.ambrosio.rodrigues@gmail.com
}

Recebido em 16/10/2012

Aceite para publicação em 16/12/2012

\section{ABSTRACT}

\section{TOOTHBRUSHING IN SCHOOL AND DENTAL CARIES: A COHORT STUDY}

Objectives: This study evaluated the role of tooth-brushing in school in reducing dental caries in childhood.

Study design: Historical cohort study

Setting: Public schools in Ovar, Portugal

Participants: Four year old children enrolled in public schools in Ovar

Methods: 62 children were divided into two groups. The first group consisted of children who brushed their teeth in school during the 21-month study period. The comparison group consisted of children who did not brush teeth at school during the same period. Data were obtained from oral health assessments conducted as part of the National Oral Health Promotion Program. We compared the number of new cavities, decayed, missing and filled deciduous teeth (dmft) and decayed, missing and filled permanent teeth (DMFT) between the two groups, after 29 months of follow-up.

Results: The risk of dental caries after 29 months was $50,0 \%$ for children who brushed their teeth at school and $62,5 \%$ for children who did not brush their teeth at school. The relative risk was 0,75 (95\% Cl: 0,42 to 1,33). The $\mathrm{dmft}$ score for children who brushed their teeth was $1,27(95 \% \mathrm{Cl}: 0,69$ to 1,94$)$ and $2,28(95 \% \mathrm{Cl}: 1,39$ to 3,19$)$ for those who did not brush $(p=0,078)$. The DMFT score was $0,37(95 \% \mathrm{Cl}: 0,08$ to 0,71$)$ for children who their brushed teeth and 0,63 (95\% Cl: 0,29-1,03) for those who did not brush $(p=0,315)$.

Discussion: No statistically significant association was found between tooth-brushing in school and reduction in the risk of dental caries, possibly because of the small sample size size.

Key Words: Toothbrushing; Dental Caries; Cohort Study. 